\title{
A Kind of Neither Keynesian Nor Neoclassical Model (5): The Path of Economic Growth
}

\author{
Zhan Zhan', Ming'an Zhan² \\ ${ }^{1}$ Westa College, Southwest University, Chongqing, China \\ ${ }^{2}$ Yunnan University, Kunming, China \\ Email:1792481782@qq.com
}

How to cite this paper: Zhan, Z. and Zhan, M.A. (2017) A Kind of Neither Keynesian Nor Neoclassical Model (5): The Path of Economic Growth. Open Access Library Journal, 4: e3525.

https://doi.org/10.4236/oalib.1103525

Received: March 15, 2017

Accepted: April 23, 2017

Published: April 26, 2017

Copyright $\odot 2017$ by authors and Open Access Library Inc.

This work is licensed under the Creative

Commons Attribution International

License (CC BY 4.0).

http://creativecommons.org/licenses/by/4.0/

(c) (i) Open Access

\begin{abstract}
Traditional macroeconomic theory is difficult to analyze the long-term growth and short-term decisions of output in a unified model. In this paper, the concept of "unit resource output" is proposed by using the difference of production factors combination on different rays in Cobb-Douglas function, and its maximization condition is derived according to algebraic principle. And then we use this condition to explain the reason why the distribution parameter $\alpha$ in the Cobb-Douglas function is growing continuously in the US statistical data and predict the evolution path of the factor combination in the growth of the output. Finally, this paper compares the important differences between our model and the Solow model.
\end{abstract}

\section{Subject Areas}

Economics

\section{Keywords}

Cobb-Douglas Function, Growth Path, Unit Resource Output

\section{Preface}

While investment and consumption growth are linked to output growth, investment and consumption are the result of output growth, not the cause [1]. Neoclassical theory regards the investment as a cause of growth, and Keynes theory turns the consumption as a cause, reversing the causal relationship between output and investment/consumption. So there are often contradictory conclusions in the neoclassical theory and Keynes theory.

In the neoclassical theory, according to the identical equation $Y=C+I_{g}+(X-M)$ and the consumption function $C=C_{a}+c Y$, when the marginal consumption coefficient $c$ is bigger, that is, the lower the savings rate $s$ 
is, the greater the multiplier effect of the investment is. That is, the smaller the investment is, the higher the output growth rate $\mathrm{d} Y / Y$ is. However, according to the result of Domar growth model $I=\theta \mathrm{e}^{\text {ast }}, Y=I / s=\theta \mathrm{e}^{a s t} / s$, where $\theta$ and $a$ are constants [2] and assume that $s$ does not change, then $\mathrm{d} Y / Y=a s \mathrm{~d} t$. This indicates that the greater the savings rate $s$ is, the higher the output growth rate $\mathrm{d} Y / Y$ is.

The Solow growth model uses $Y=A K^{\alpha} L^{\beta}$ as the production function, and also uses the assumption $Y=I / s$ as Domar model that "the smaller $s$ is the greater the output is". According to the result of the Solow model

$\mathrm{d} k=s A k^{\alpha}-\lambda k \quad$ (where $\left.k=K / L\right)$ [3], from $y=A k^{\alpha} \quad$ (where $\left.y=Y / L\right)$, then $\mathrm{d} y=\alpha A k^{\alpha-1} \mathrm{~d} k=\alpha A k^{\alpha-1}\left(s A k^{\alpha}-\lambda k\right), \mathrm{d} y / y=\alpha\left(s A k^{\alpha-1}-\lambda\right)$. This shows that only a higher savings rate of $s$ could delay the appearance of $\mathrm{d} Y / Y=0$.

The Solow model looks more comprehensive than the Domar model, and its conclusion that economic growth will eventually stop is more surprising, thus stimulating more research enthusiasm. On the one hand, more and more statistical data show that, for a long period of time, although the per capita output of different countries are quite different, but the per capita real output growth rate did not significantly decline [4]-[10]. On the other hand, people improves to the limitations of "exogenous growth theory" in the Solo model, or to reset the production function [11] [12] [13], or endow new meanings for production factors in the Cobb-Douglas function.

In our growth model, the reasons for the growth of output, whether short or long, are the same. The difference is only: in the short term, in addition to growth but also to analyze the growth of the fluctuations [14] [15] [16]. In the long term, on the basis of growth should also analyze the growth of the path characteristics.

\section{Comparison of Unit Resource Output}

In analyzing the employment problem, we have assumed that the material resource $K$ and the human resource $L$ have the same dimension and associate $K$ and $L$ using the inequality in the Cobb-Douglas function [14]:

$$
Y=A K^{\alpha} L^{\beta} \leq A \frac{\left(K^{\alpha}\right)^{2}+\left(L^{\beta}\right)^{2}}{2}
$$

Among them, the conditions to reach the upper boundary are:

$$
L^{\beta}=K^{\alpha} \rightarrow L=K^{\alpha / \beta}
$$

If $\beta$ is larger, the output (income) $Y$ will be more allocated to the production factor $L$, at the same time, by $L=K^{\alpha / \beta}$ we can see that the value of $K$ and $L$ will be relative changes. In the Cobb-Douglas function $Y=A K^{\alpha} L^{\beta}, \alpha+\beta=1$, by the Euler theorem [17], the corresponding constraint equation or the equal-cost line is:

$$
Y=\frac{\partial Y}{\partial K} K+\frac{\partial Y}{\partial K} L
$$

Let $\partial Y / \partial K=r, \partial Y / \partial L=w$, then 


$$
Y=r K+w L
$$

Assuming $\alpha / \beta<1$, then the equal-output lines and the equal-cost lines of $Y=A K^{\alpha} L^{\beta}$ are shown in Figure 1 .

Because $Y_{2}>Y_{1}$, the point $y_{2 a}$ is on the equal-output line $Y_{2}$, the point $y_{1 a}$ is on the equal-output line $Y_{1}$, so the output of the point $y_{2 a}$ in the graph is larger than the point $y_{1 a}$. On the curve $Y_{1}$, the outputs of points $y_{1 a}$ and points $y_{1 b}$ are the same, but their factor combinations $(K, L)$ are different. We now want to prove that the production state of point $y_{1 a}$ is better than that of point $y_{1 b}$ : the consumption $\left(K_{1 a}+L_{1 a}\right)$ of the two resources at point $y_{1 a}$ is less than $\left(K_{1 b}+L_{1 b}\right)$ of point $y_{1 b}$. Due to

$$
\frac{\partial Y}{\partial K}=\frac{\alpha Y}{K}=r, \frac{\partial Y}{\partial L}=\frac{\beta Y}{L}=w,
$$

So

$$
K=\frac{\alpha Y}{r}, L=\frac{\beta Y}{w}
$$

From the algebraic inequality $a+b \geq 2 a^{1 / 2} b^{1 / 2}$, when $a=b, a+b=2 a^{1 / 2} b^{1 / 2}$, and so

$$
K+L=\frac{\alpha Y}{r}+\frac{\beta Y}{w} \geq 2 Y \cdot \frac{\alpha^{1 / 2} \beta^{1 / 2}}{r^{1 / 2} w^{1 / 2}}
$$

at the point $y_{1 b}$, because $r_{y_{1 b}} \neq w_{y_{1 b}}$, so

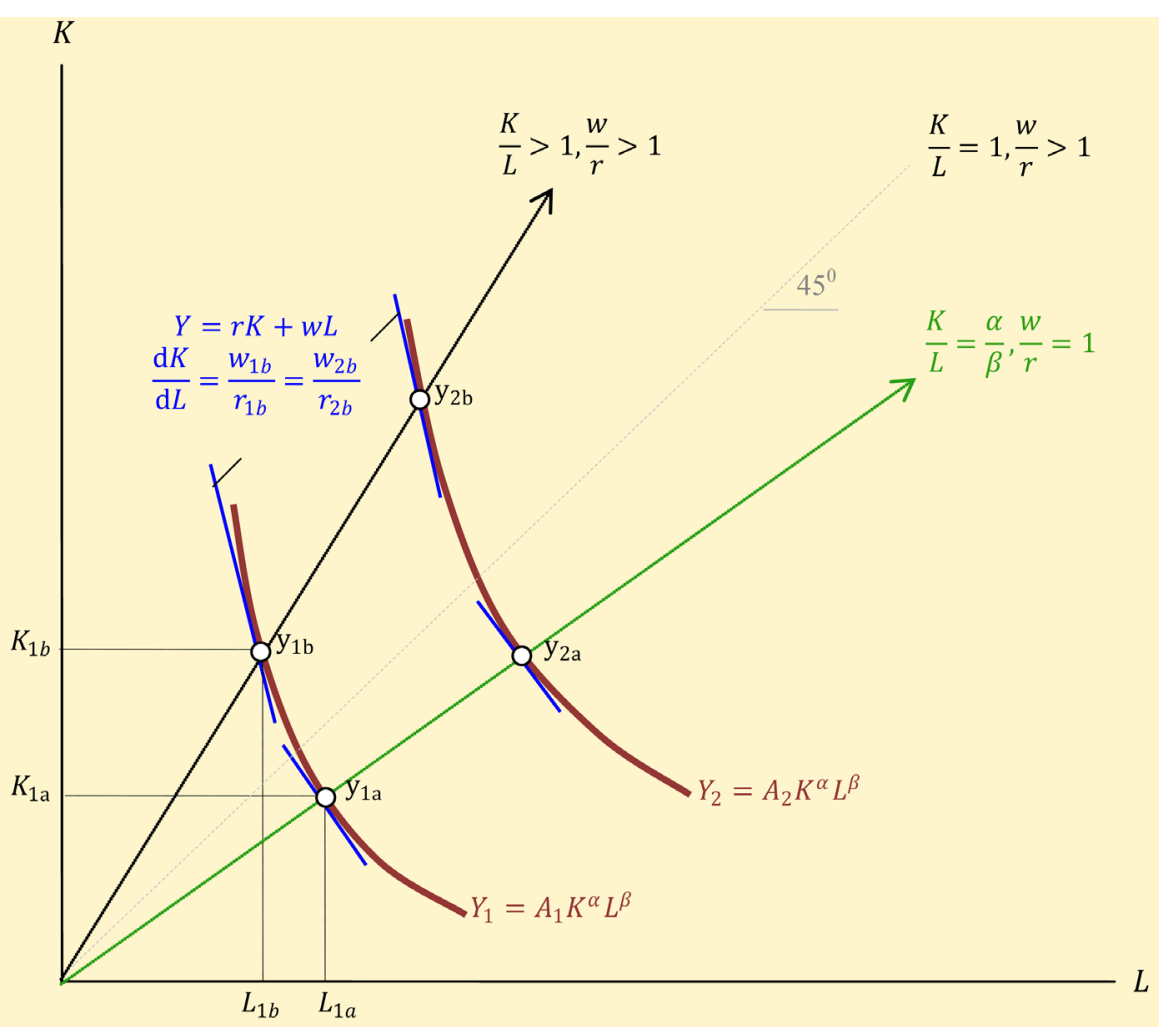

Figure 1. "Unit resource output" comparison. Note: This figure is not from the statistical data, which is the schematic diagram of the Cobb-Douglas function $Y=A K^{\alpha} L^{\beta}$, where the equal-output lines are roughly drawn according to the characteristics of $\alpha / \beta<1$. 


$$
K_{1 b}+L_{1 b}=Y\left(\frac{\alpha}{r_{1 b}}+\frac{\beta}{w_{1 b}}\right)>2 Y \cdot \frac{\sqrt{\alpha \beta}}{\sqrt{r_{1 b} w_{1 b}}}
$$

at the point $y_{1 a}, \quad r_{y_{1 a}}=w_{y_{1 a}}$, so

$$
K_{1 a}+L_{1 a}=Y\left(\frac{\alpha}{r_{1 a}}+\frac{\beta}{w_{1 a}}\right)=2 Y \cdot \frac{\sqrt{\alpha \beta}}{\sqrt{r_{1 a} w_{1 a}}}
$$

To prove that $K_{y_{1 b}}+L_{y_{1 b}}>K_{y_{1 a}}+L_{y_{1 a}}$, we also need to prove that $\sqrt{r_{1 b} w_{1 b}}=\sqrt{r_{1 a} w_{1 a}}$, or $\sqrt{r_{1 b} w_{1 b}}<\sqrt{r_{1 a} w_{1 a}}$. According to Equation (4) $Y=r K+w L, \quad r K+w L \geq 2 \sqrt{r w} \sqrt{K L}, \quad Y \geq 2 \sqrt{r w} \cdot \sqrt{K L}$, so $\sqrt{r w} \leq Y / 2 \sqrt{K L}$. By Equation (2) $L=K^{\alpha / \beta}$,

$$
\frac{Y}{2 K^{1 / 2 \beta}} \geq \sqrt{r w}
$$

at the point $y_{1 b}$, because $r_{y_{1 b}} \neq w_{y_{1 b}}$, so

$$
\frac{Y}{2 K_{1 b}^{1 / 2 \beta}} \geq \sqrt{r_{1 b} w_{1 b}}
$$

at the point $y_{1 a}, r_{y_{1 a}}=w_{y_{1 a}}$, so

$$
\frac{Y}{2 K_{1 a}^{1 / 2 \beta}}=\sqrt{r_{1 a} w_{1 a}}
$$

Compare $K_{1 a}$ on the point $y_{1 a}$ and $K_{1 b}$ on the point $y_{1 b}, K_{1 a}<K_{1 b}$, so,

$$
\frac{Y}{2 K_{1 a}^{1 / 2 \beta}}>\frac{Y}{2 K_{1 b}^{1 / 2 \beta}}
$$

so

$$
\sqrt{r_{1 a} w_{1 a}}>\sqrt{r_{1 b} w_{1 b}}
$$

Back to Equation (7) (8), so,

$$
K_{1 b}+L_{1 b}>K_{1 a}+L_{1 a}
$$

Although the output at the point $y_{1 b}$ and the point $y_{1 a}$ is the same, the total amount of resources required by the former is greater than the latter, or the former unit resource output is less than the latter, that is,

$$
\frac{Y_{1}}{K_{1 b}+L_{1 b}}<\frac{Y_{1}}{K_{1 a}+L_{1 a}}
$$

This proves that the closer to the marginal output $\partial Y / \partial K$ and $\partial Y / \partial L$ on the same production line, the greater the unit resource output. In the process of output growth, the Cobb-Douglas function $Y=A K^{\alpha} L^{\beta}$ changes at any time because $\alpha / \beta$ is always changing. In general, the factor combination $(L, K)$ is closer to the ray $w / r=1$, the greater the unit resource output. When $\alpha / \beta=1$, the factor combination on the bisector line $K=L$ will be the best use of resources.

\section{The Path of Economic Growth}

In discussing the relationship between variables in the medium and short-term relationship, we always assume that $\alpha$ or $\beta$ is constant. The changes of $\alpha$ in the 
medium and short-term is indeed small (in 1970 and 2016, the United States $\alpha$ were 0.2770 and 0.3691 respectively), but in the long-term, the changes of $\alpha$ can not only be ignored, but also an important variable in the analysis of the path of output growth.

As Figure 2 shows, $\alpha$ in the United States there are two kinds of statistical data changes, first is the short-term fluctuations, the second is the long-term trend of change. In the discussion of short-term problems, we always assume $\mathrm{d} \alpha / \alpha=0$. Therefore, although the periodic equations describe the relationships between the main variables, there are always some deviations when using statistical data to verify them.

In both sides of the Equation (2) $L^{\beta}=K^{\alpha}$ take the logarithm, and the use of the equation $\alpha+\beta=1$, we can get:

$$
\frac{\beta}{\alpha}=\frac{\ln K}{\ln L}, \alpha=\frac{\ln L}{\ln K+\ln L}, \mathrm{~d} \alpha=\frac{\beta \cdot \frac{\mathrm{d} L}{L}-\alpha \cdot \frac{\mathrm{d} K}{K}}{\ln K+\ln L}
$$

so

$$
\frac{\mathrm{d} \alpha}{\alpha}=\frac{\beta \cdot \frac{\mathrm{d} L}{L}-\alpha \cdot \frac{\mathrm{d} K}{K}}{\ln L}
$$

This indicates that, as the output grows, $L \rightarrow K,(\beta \mathrm{d} L / L-\alpha \mathrm{d} K / K) \rightarrow 0$, $\mathrm{d} \alpha / \alpha \rightarrow 0$. Theoretically, the growth of $\alpha$ is the marginal decline, that is, the growth rate of $\alpha$ in the future will be slower and slower. In Figure 2, in addition to the trend line (gray) that is automatically generated by Office Excel, we also draw a red curve manually to indicate the trend of decreasing $\alpha$ growth.

If the result of the increment of $\alpha$ is 1, it means that all the output $Y$ will be assigned to the capital factor $K$, since the marginal contribution of $K$ is infinite. This state is very puzzling because the labor factor $L$ is infinite, but its marginal contribution is 0 .

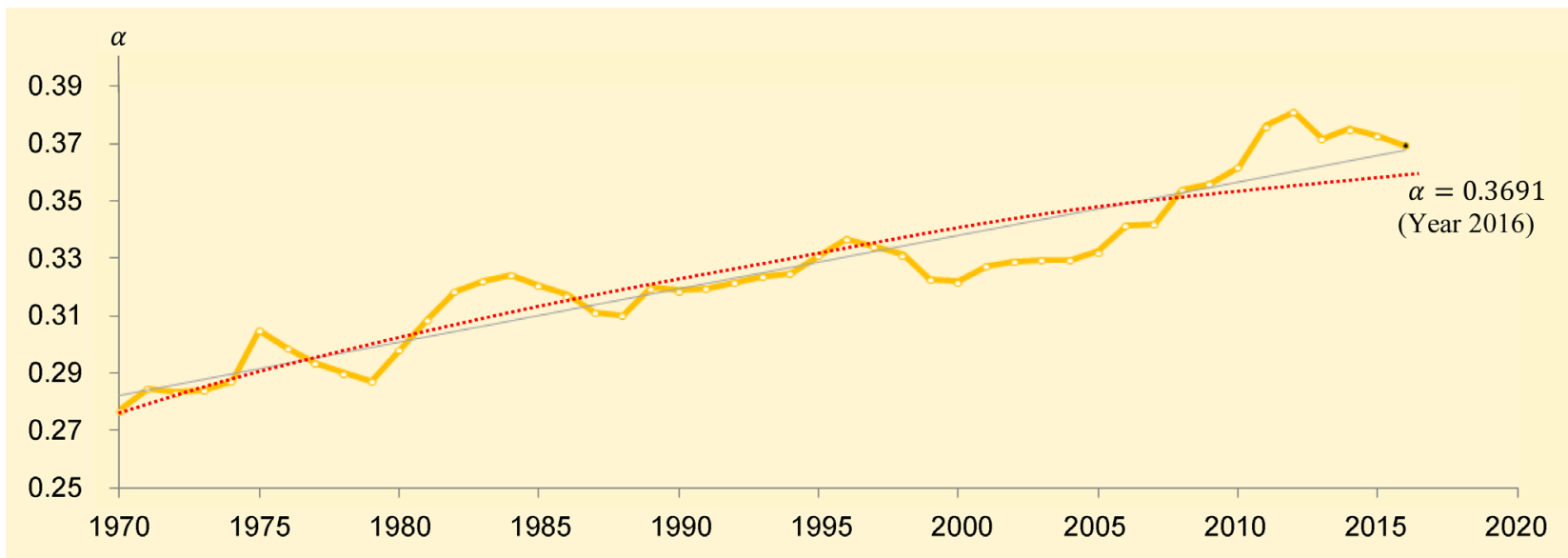

Figure 2. Trend of change of $\alpha$. Source: 1) $\alpha=1-\beta, \beta$ is the share of salary income in GDP, and the data for salary income and GDP are derived from Table $2.1 \mathrm{in} \mathrm{http://www.bea.gov/.2)} \mathrm{The} \mathrm{gray} \mathrm{line} \mathrm{is} \mathrm{the} \mathrm{trend} \mathrm{line} \mathrm{that} \mathrm{is} \mathrm{automatically} \mathrm{generated}$ by Office Excel. The red dotted curve is manually drawn to indicate the characteristic of growth marginal decreasing of $\alpha$. 
Since the marginal output of $L$ is zero, why do we not reduce the amount of $L$ and increase the amount of $K$, so that $\partial Y / \partial K=\partial Y / \partial L$ ? Thus $\alpha=1$ is an uneconomical state.

If the ending state of $\alpha$ is $1 / 2$, then $\alpha / \beta=1$. From the Equation (2) $L^{\beta}=K^{\alpha}$, when $\alpha / \beta \rightarrow 1, K / L \rightarrow 1$, so

$$
r=\frac{\partial Y}{\partial K}=\frac{\alpha Y}{K}=\frac{\beta Y}{L}=\frac{\partial Y}{\partial L}=w
$$

Although $L^{\beta}=K^{\alpha}$ is the optimal state of the Cobb-Douglas function $Y=A K^{\alpha} L^{\beta}$ to its geometric boundary, it is only the "suboptimal state". It is necessary to further satisfy the marginal state of $\partial Y / \partial K=\partial Y / \partial L$, which is the "optimal state" of the Cobb-Douglas function under certain output, that is, the greatest state of "unit of resources output".

According to the prediction of $\alpha / \beta \rightarrow 1$, Figure 3 shows the schematic diagram of the output from $Y_{1}$ to $Y_{n}$ (red dotted lines of connection points $y_{1 b}$ and $y_{n b}$ in the figure). Since $\alpha$ is constantly changing during the growth of $Y$, the Cobb-Douglas function $Y=A K^{\alpha} L^{\beta}$ is also changing. As the growth path shown by the red dotted line gradually approaches the bisector $K / L=1$, the ray satisfying the condition $w=r$ is also moving closer to the bisector $K / L=1$.

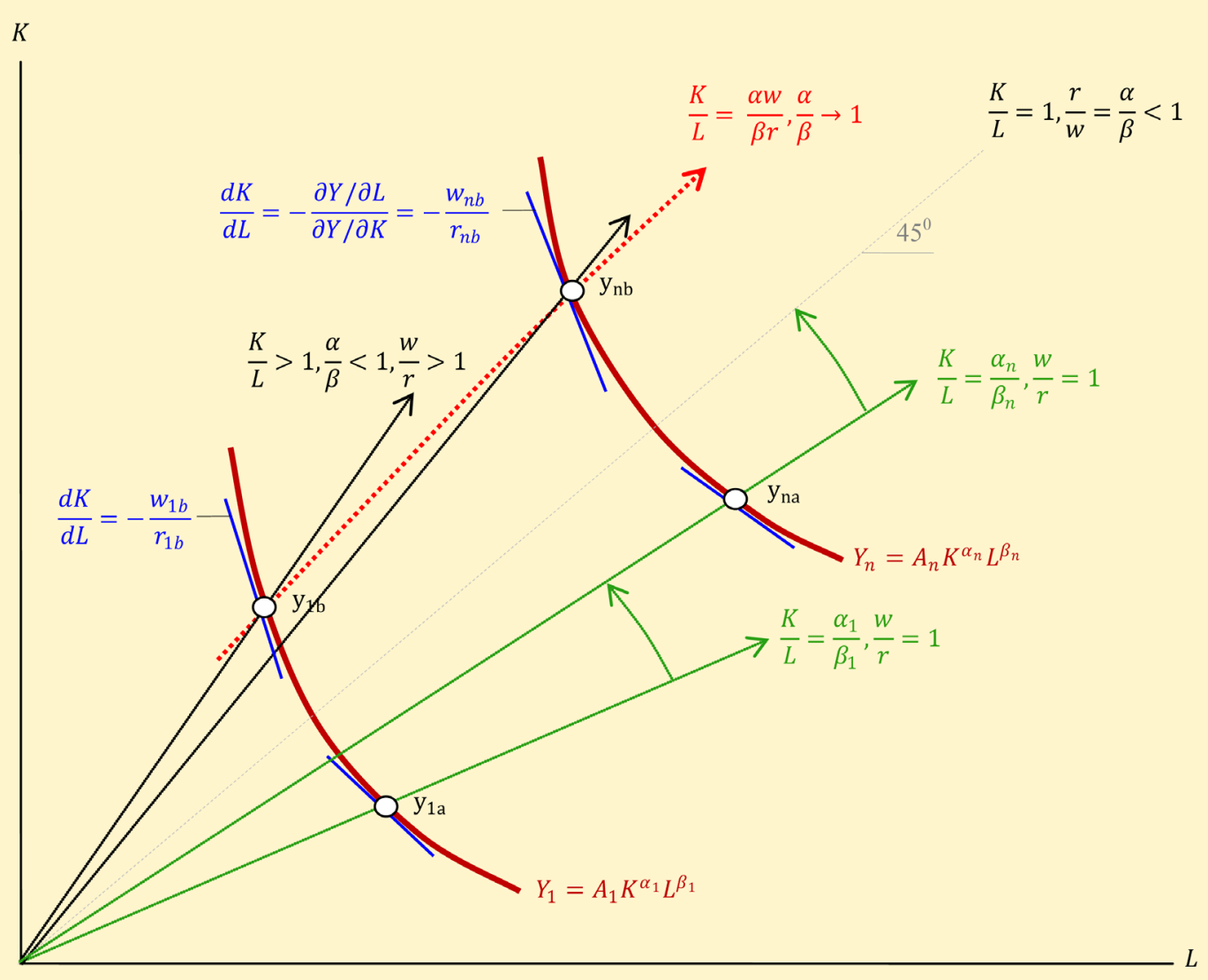

Figure 3. The principle of the output growth path. Note: 1) The red dotted curve in the figure is the change path of the factor combination in the output growth. 2) The ray satisfying the $w / r=1$ will be close to the bisector $K / L=1$ with $\alpha / \beta \rightarrow 1$. 
The Cobb-Douglas function $Y=A K^{\alpha} L^{\beta}$ will eventually evolve into the function $Y=A K^{1 / 2} L^{1 / 2}$ for the bisector $K / L=1$ symmetry.

Figure 4 shows the growth path of GDP in US statistics. Although the human resource factor $L$ is increasing with the increase of $\alpha$ according to the definition of the formula $L^{\beta}=K^{\alpha}$, it is still small relative to the factor $K$ (in 2016, $K=$ USD 535,421.8 billion, $L=$ USD 2247.9 billion). In order to reflect the relative position of the growth path of $Y$ in the statistical data and the bisector $K=L$, we use the logarithmic coordinates in the main graph. As shown in Figure 3, the growth path of $Y$ in Figure 4 is indeed on the left side of the bisector $K=L$, and there is a trend toward the bisector $K=L$.

The economic meaning of the production status on the right side of the bisection line is: in the price of resource, the labor factor is higher than the capital factor $(w>r)$; in the distribution of output, the proportion of labor factor is smaller than that of capital factor $(\beta<\alpha)$.
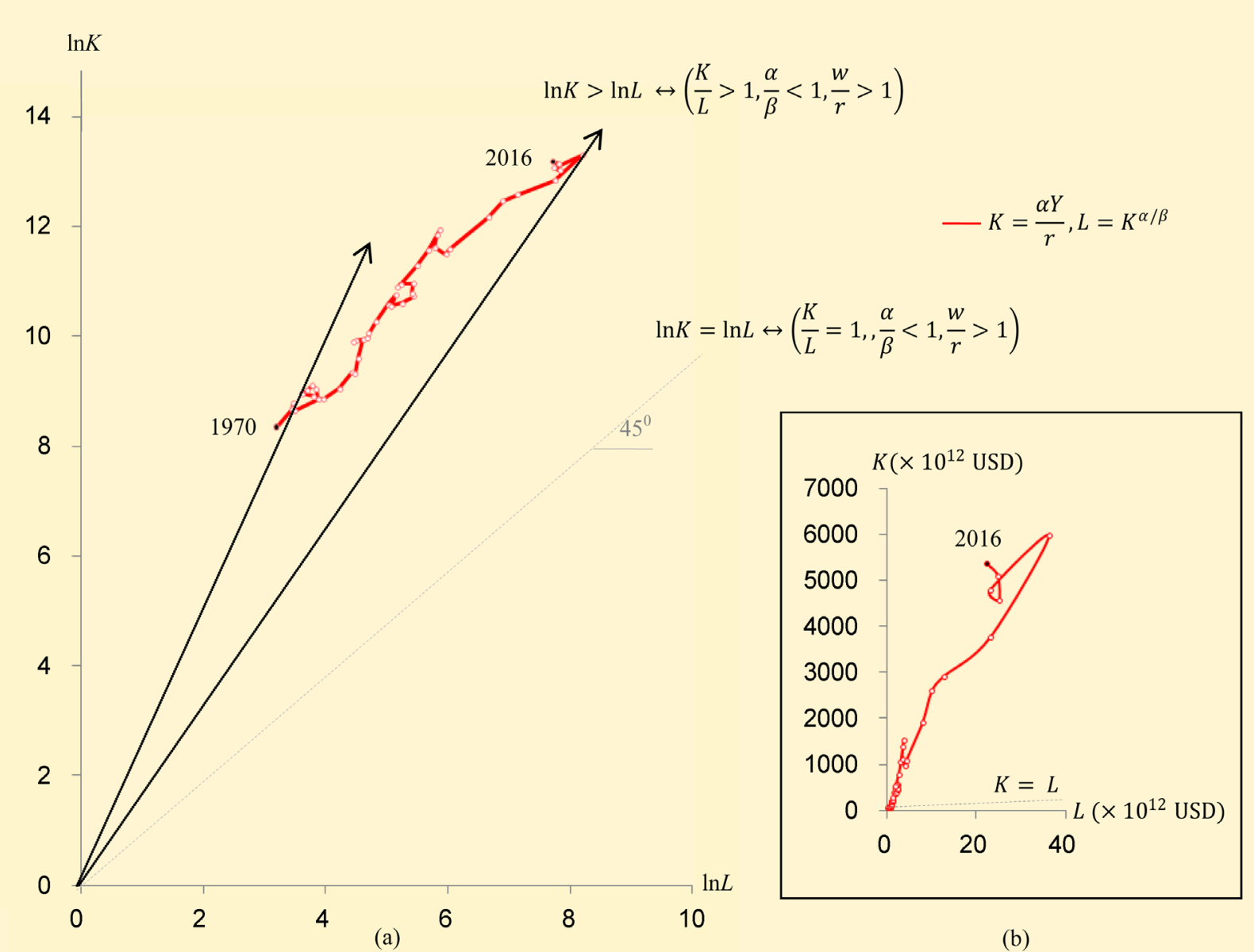

(b)

Figure 4. The growth path from the statistical data. Source: 1) Since the gap between $K$ and $L$ is large, Figure 4(a) uses logarithmic coordinates to observe the relative position of the growth path with the bisector $K / L=1.2$ ) Figure $4(\mathrm{~b})$ is not the logarithmic coordinates, $K=\alpha Y / r, L=K^{\alpha / \beta}$. The data for $\alpha$ is the same as Figure 2. $Y=$ GDP, GDP data comes from Table 1.1.5 in http://www.bea.gov/. The interest rate $r$ is the average of the annualized yields of various types of US Treasury bonds, and the original data is from http://www.federalreserve.gov/. The calculation method is described in the paper "A Kind of not Keynesian nor Neoclassical Model (2): The Business Cycle" [17]. 
This explains why the growth path starts on the left side of the bisector $K=L$, not the right side: When the level of output is low, or if the lower output is sufficient to maintain the basic living of the worker, the income should be preferentially assigned to the factor $L$, irrespective of the marginal output of $K$. If the income of workers cannot feed themselves and their families, production activities cannot be carried out.

With the economic growth, the basic survival needs of the factors of labor $L$ need to be met, the factors elements $L$ and $K$ will compete in production. As long as $\partial Y / \partial K \neq \partial Y / \partial L$, the use of one of the factors is more efficient than the other, so $\alpha$ rises continuously until $\alpha / \beta=1$. This is why the distribution coefficient $\alpha$ is increasing in US statistics.

From a macro-field of view, $\alpha=\beta=1 / 2$ is the total output $Y$ by the capital factor $K$ and labor factor $L$ equally. If the micro-field is the case, it means that half of everyone's income from investment, the other half from wages. Perhaps, the proper work helps to avoid the spirit of emptiness when doing nothing, so $\alpha=\beta=1 / 2$ is a "happiness" way of life.

Unfortunately, we cannot prove that $K$ and $L$ are equal to the macro-income structure of $Y$, will produce the same micro-income structure. In the macroeconomic $\alpha=\beta$, There may be a situation in which the income of some of the total population comes from capital, and the income of the other part comes from wages. This means that everyone is happy in society, as a whole is also happy, but the overall happiness of the community is not necessarily everyone happy.

\section{The Path of the Traditional Growth Model}

$L$ in the Cobb-Douglas function is assumed to be the labor force in the Solow model, so that $Y=A K^{\alpha} L^{\beta}$ can be reduced to the unary function $y=A k^{\alpha}$, where $y=Y / L, k=K / L$. In fact, from the mathematical point of view, $L$ does not have to be assumed to be the labor force, even if $L$ is the population of children, so that $Y=A K^{\alpha} L^{\beta}$ can be transformed into $y=A k^{\alpha}$.

For the unary function $y=A k^{\alpha}$, if $A$ is constant, then

$$
\frac{\mathrm{d} y}{\mathrm{~d} k}=\frac{\alpha A}{k^{\beta}} \rightarrow 0 \text {, when } k \rightarrow \infty
$$

So, as long as the $A$ in the Cobb-Douglas function is constant, the growth rate of $y$ will become slower and slower without the constraints such as $\Delta K=I$ and $I=s Y$ are assumed in the Solow model, as the red curve shows in Figure 5.

On the other hand, in $Y=A K^{\alpha} L^{\beta}$, when $\partial Y / \partial K=r$, then the marginal state equation $Y=r K / \alpha$. In the equation $Y=r K / \alpha$, when both sides divided by $L(\neq 0)$, we can get $y=(r / \alpha) k$. This means that when $r / \alpha$ is constant, $y$ and $k$ are linearly related, as the blue line showed in Figure 5 . The reason for this contradiction comes from the consideration of $A$ in $y=A k^{\alpha}$ as an exogenous variable. If $A$ grows also as $k$ grows, we can explain the relationship between $y=A k^{\alpha}$ and $y=(r / \alpha) k$ as shown in Figure 5 .

In Figure 5, suppose point a is the initial state of output. When $k$ increases from $k_{1}$ to $k_{2}$, if $A$ does not change, point a will move to point $\mathrm{c}$ along the 


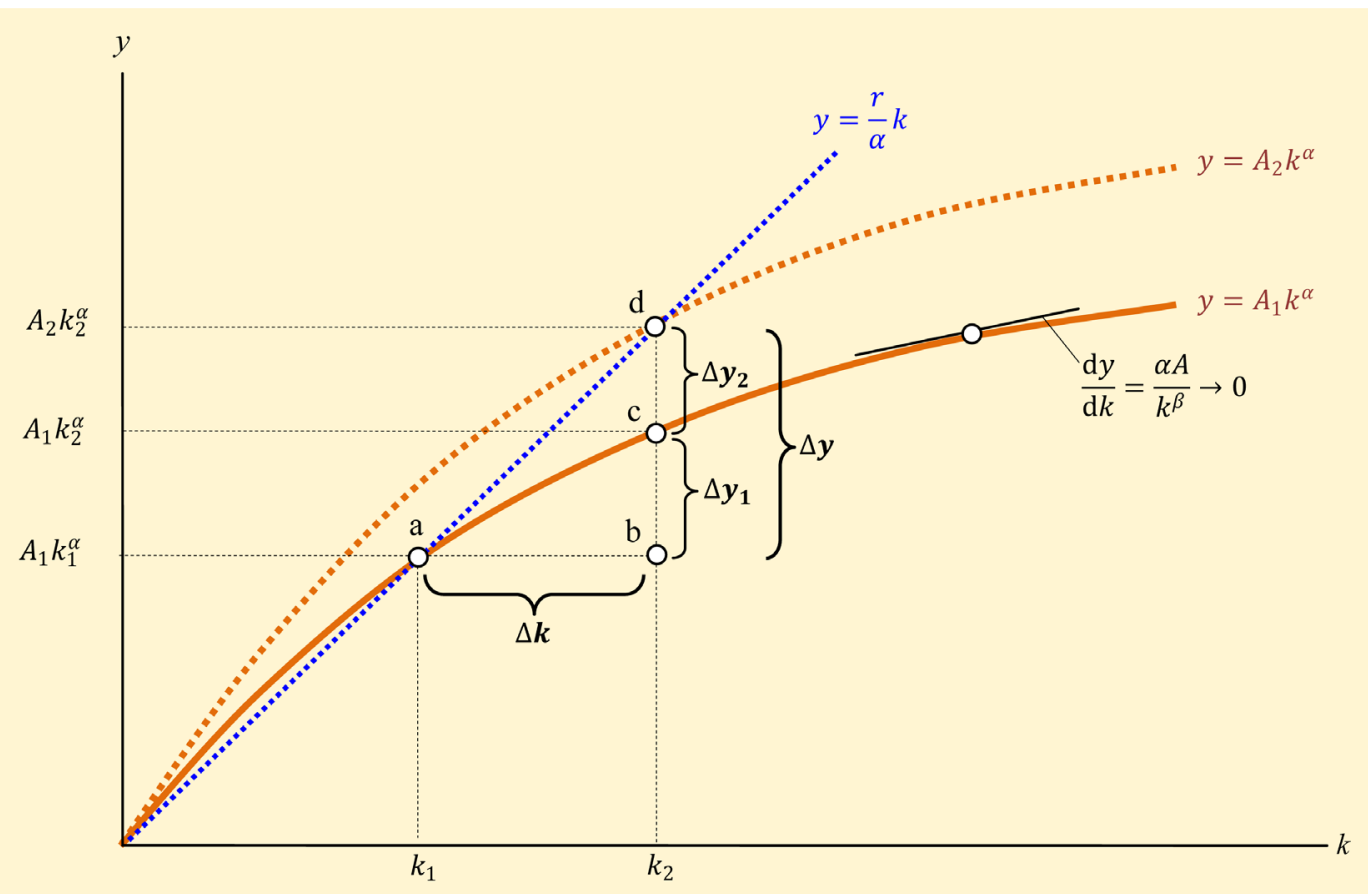

Figure 5. The reason that $y / k$ does not decline. Notes: 1) $y=A k^{\alpha}$ generates from $Y=A K^{\alpha} L^{\beta}$, in which $y=Y / L, k=K / L$. 2) $y=(r / \alpha) k$ generates from $Y=(r / \alpha) K$, in which $r=\partial Y / \partial K=\partial\left(A K^{\alpha} L^{\beta}\right) / \partial K=\alpha Y / K, \quad y=Y / L, k=K / L$.

function $y=A_{1} k^{\alpha}$. If $A$ increases from $A_{1}$ to $A_{2}$ while $k$ changes, the result of the change is point $\mathrm{d}$. This is exactly the output of the marginal state equation $y=(r / \alpha) k$ of the Cobb-Douglas function.

The Domar model assumes that the production function is $Y=a K$ [2]. In the equation on both sides divided by $L$, then $y=a k$. This is similar to the marginal state equation $y=(r / \alpha) k$. From this point of view, the Domar model is not unreasonable, but, as the Solow model, since it further assumes that $\Delta K=I, I=s Y$, the so-called "knife-edge" problem is encountered in discussing the balance between investment demand and investment supply.

As with the Solow model, if we further assume constraints such as $\Delta K=I$ and $I=s Y$, the growth of $y$ is affected not only by the diminishing marginal output of $k$ but also by the depreciation of the capital, so $y$ is will stop growing in a finite time. Our analysis shows that capital depreciation is simply not a constraint on output growth, since $\Delta K$ is much larger than $I$, and as long as $r>0$, the growth of $y$ does not stop [1].

Since the $\alpha / \beta$ in the Solow model does not change, in the coordinates of $K$ and $L$, the equal output line of $Y$ should be the same family curve of CobbDouglas function. Because $K$ is the number of money, $N$ is the number of people, so their numbers are not comparable. But if we choose the appropriate dimension, such as the dimension of 1000 billion USD for $K$, and the dimension of 10,000 thousands of people for $N$, we can also have a bisector of $K=N$. This can be compared with the growth path of output $Y$ in the Solow model as showed in Figure 4. 
As shown in Figure 6, in order to reflect the relative position of the growth path of the output $Y$ in the statistical data and the bisector $K=N$, we use the logarithmic coordinates in the main picture as shown in Figure 4.

According to this dimension, when the output level is low, $K<N$, the factors combination point is below the bisector $K=N$. Since the growth of $K$ is much faster than the growth of $N$, with the increase in the level of output, the factors combination would soon pass through the bisector to the top of the bisector from below the bisector (about Year 1980).

Since $\partial Y / \partial K=\alpha Y / K, \partial Y / \partial N=\alpha Y / N$, make $\partial Y / \partial K=r, \partial Y / \partial N=w$, then $w / r=\beta / \alpha$ on the bisector $K=N$. Since $\alpha / \beta<1$ in the statistical data, then $w / r>1$, and $w / r$ increases when $K / N$ increases, therefore the marginal output gap between $K$ and $N$ is increasing. This is not as shown in Figure $4, w / r \rightarrow 1$ due to the decrease of $K / L$. So, in the economic sense, the growth path of Solow model does not tend to be a better marginal state. This has nothing to do with the dimension setting of $K$ and $N$.

The above analysis shows that although the Solow model and the model we use in this paper both use the Cobb-Douglas function to analyze the output

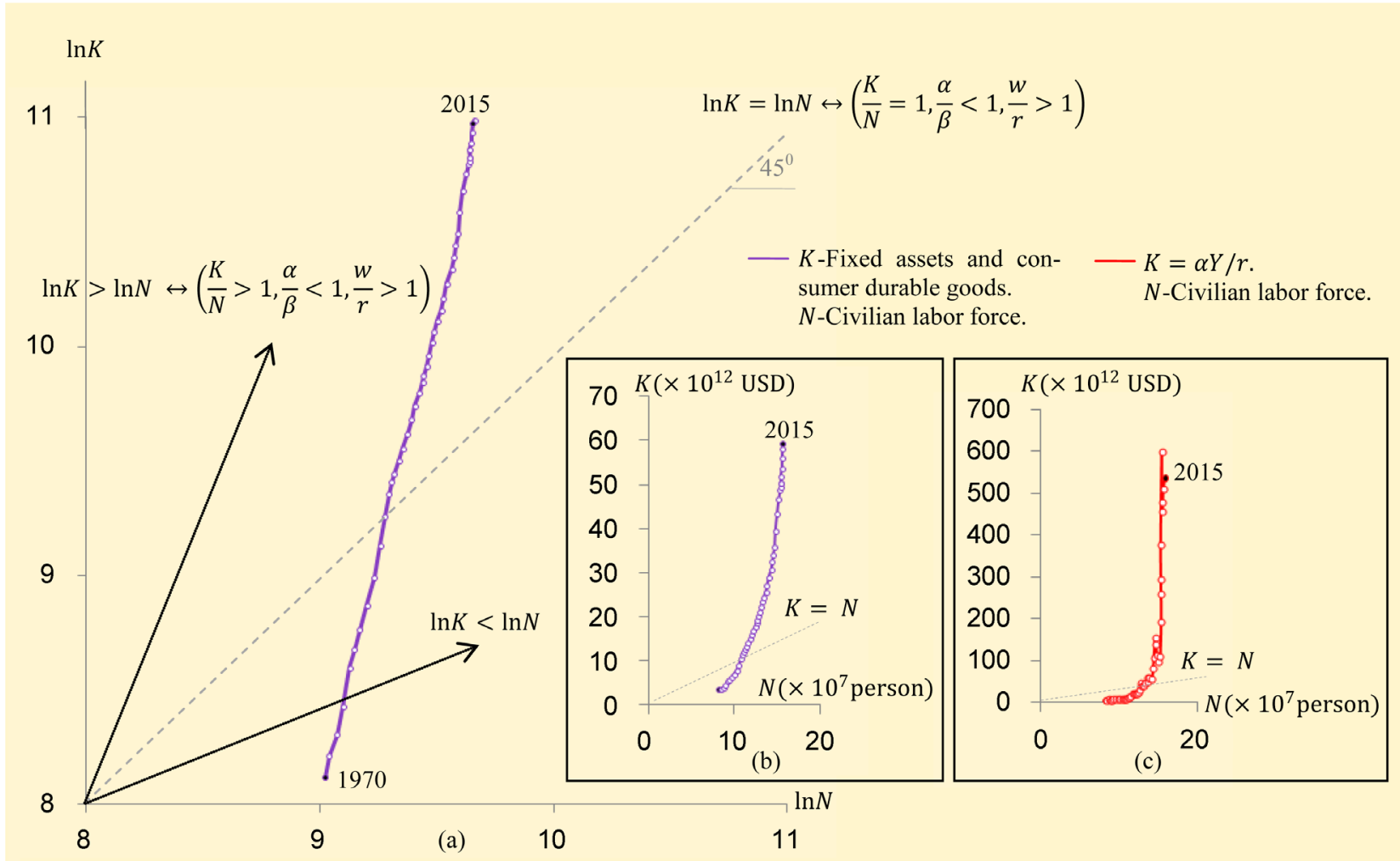

Figure 6. Grow path of Solow model in statistics. Resources: 1) Since the variable $N$ is much smaller than the figure of $K$, in order to reflect the relative position of the growth path of the output in the statistical data and the bisector $K / N=1$ we used the logarithmic coordinates in Figure 6(a). 2) Figure 6(b) use non-logarithmic coordinates, in which, since Solow model does not relate $Y=A K^{\alpha} L^{\beta}$ to $K=\alpha Y / r$, date of $K$ use "Fixed assets and consumer durable goods", sources are from http://www.bea.gov/ Table 1.1. (by March 2017, there was no statistical data for "Fixed assets and consumer durable goods" in 2016). Date of $N$ is "Civilian labor force", sources are from http://www.bls.gov/cps/cpsaat01. 3) $K$ in Figure 6(c) is calculated by the function (5) $K=\alpha Y / r$ so as to be compared with Figure 4(b). The gap between $K$ and $N$ is larger because the calculated $K$ is much larger than "Fixed assets". This indicates that no matter what dimension and which kind of $K$, the difference between the marginal variablewand $r$ will be larger and larger. 
growth, there are three differences: First, the Solow model does not regard the conditional parameter $A$ as a variable. Second, the Solow model ignores the importance of change of $\alpha / \beta$. Third, the Solow model assumes that the factor $L$ in the Cobb-Douglas function is the labor force $N$.

In this model, we can see from Equation (9) that $A$ is an endogenous variable that contains the marginal state of production and cannot be assumed to be an arbitrary constant. In the short-term analysis, we always ignore the change of the distribution of parameters $\alpha / \beta$, but in the long-term, we need to explain its trend of change, and it is an essential link when we discuss the marginal relationship between production factor $K$ and $L$. Although it can be assumed mathematically that $L=N$, the above analysis shows that this hypothesis does not help us to analyze the relationship between macroscopic variables.

In practice, we can denote $Y=A K^{\alpha} L^{\beta}$ into a unary function $y=A k^{\alpha}$ under the condition of $y=Y / L, k=K / L$ and $L \neq 0$ without assuming $L=N$. If $L$ is defined by Equation (2) $L=K^{\alpha / \beta}$, then $L$ will increase when $K$ increases. Since the growth rate of $\alpha / \beta \rightarrow 1, L$ will increase more than $K$, therefore, when $K / L>1, k=K / L$ and $y=Y / L$ do not rise but decrease.

The point $y_{1 b}$ in Figure 7 corresponds to the point $y_{1 b}$ in Figure 3. Although at the point $y_{1 b}, K$ and $Y$ are smaller, $y$ and $k$ larger due to the small $L$. Since $\alpha / \beta<1, L$ increase faster than that of $Y$ and $K$, therefore $y$ and $k$ decrease. On the time path, $y=A k^{\alpha}$ moves from the point $y_{1 b}$ to $K / L=1$.

Is there a situation where $\alpha / \beta>1$ when the level of output is low in reality? That is, where is the share of $K$ from the output higher than $L$ ? This is likely to

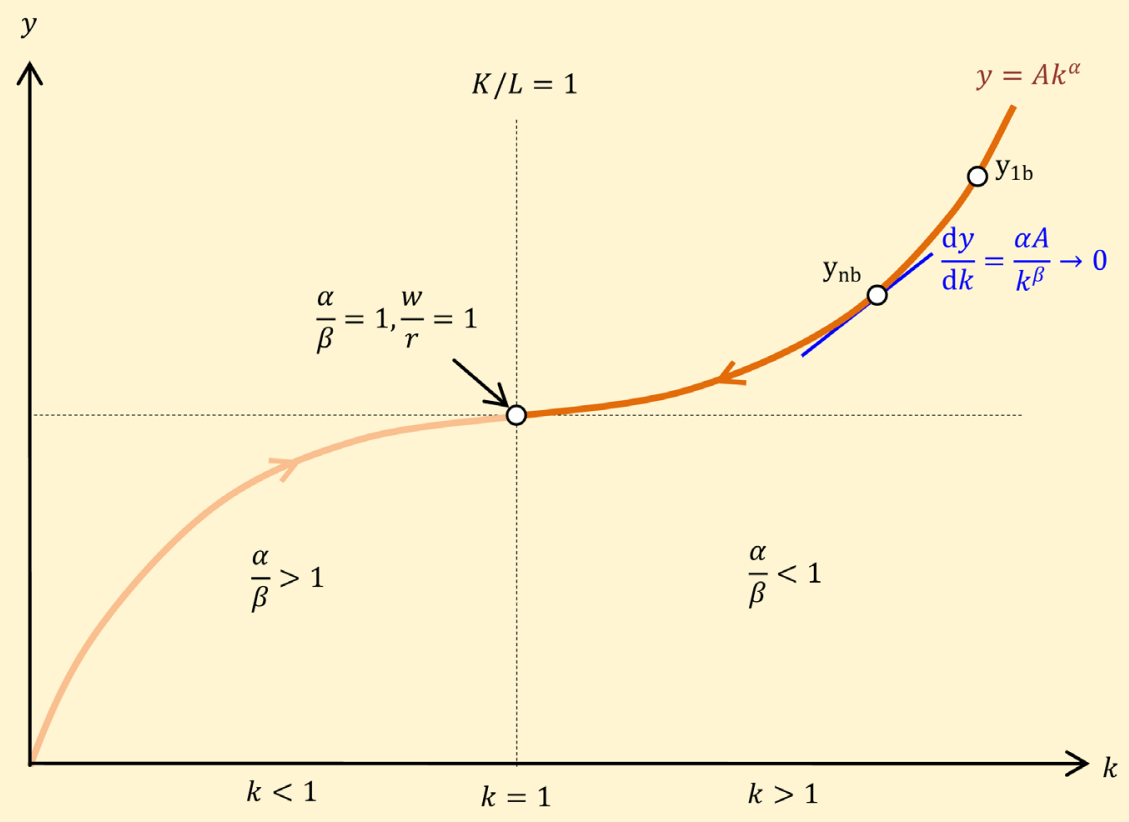

Figure 7. The growth path in the coordinates $(k, y)$. Notes: 1) $y=A k^{\alpha}$ is from $Y=A K^{\alpha} L^{\beta}$, in which $K=\alpha Y / r, L=K^{\alpha / \beta}$, $y=Y / L, k=K / L .2)$ According to $L^{\beta}=K^{\alpha}$, when $\alpha / \beta<1, K>L, k>1$; when $\left.\alpha / \beta>1, k<1.3\right)$ In the increasing process of $Y, \alpha / \beta \rightarrow 1$, so $k=K / L \rightarrow 1, \quad r / w=(\alpha Y / K) /(\beta Y / L) \rightarrow 1$. 
occur in countries that are technically low level and a large number of foreign advanced technologies and capital are introduced. At this point, the combination of the factors $K$ and $L$ will approach the bisector from the right side of the bisector $K=L$ in Figure 3. Corresponding to this, $y=A k^{\alpha}$ in Figure 7 approaches $k=1$ from $k<1$.

\section{Summary}

\subsection{Assumptions}

- The production function in the market economy system: $Y=A K^{\alpha} L^{\beta}$, Marginal conditions: $r=\partial Y / \partial K=\alpha Y / K, \quad w=\partial Y / \partial L=\beta Y / L$.

- $L^{\beta}=K^{\alpha}$ or $=K^{\alpha / \beta}$.

- $\partial Y / \partial K=\partial Y / \partial L$ when $t \rightarrow \infty$.

\subsection{Outcome}

- On the same line of production, "unit resource output" is the largest on $r=w$ ray.

- The path of output growth is formed in the process of equalizing the marginal output of various factors to each other.

\section{Discussions}

The fundamental cause of the contradiction between the conclusions and assumptions of the Solow model is that some of the assumptions (including implicit assumptions) are erroneous. We have analyzed the existence of the CobbDouglas function $Y=A K^{\alpha} L^{\beta}$ in the "A Kind of Neither Keynesian Nor Neoclassical Model (1): The Fundamental Equation", which has implied the output is growing in an economic system. Because in $Y=A K^{\alpha} L^{\beta}$, the distribution coefficient $\alpha=[(\partial Y / \partial K) K] / Y$, only when $\partial Y / \partial K>0$, then $\alpha>0$, and thus Cobb-Douglas function is significative. This paper simply expresses the implied growth state of $Y=A K^{\alpha} L^{\beta}$ in a better understanding way. So our output decision model is not logically contradictory both in short-term and long-term.

Figure 3 and Figure 7 show that with $\alpha / \beta \rightarrow 1$, the output growth would face some sort of the ending state, although in time this process may be infinite. This result is generated when we assume $L=K^{\alpha / \beta}$, and if we assume different properties for human resource factor $L$, as in many other endogenous growth models [18] [19], will there be any difference? We will discuss further in the follow-up paper.

\section{References}

[1] Zhan, M.A. and Zhan, Z. (2016) A Kind of Neither Keynesian Nor Neoclassical Model (1): The Fundamental Equation. Open Access Library Journal, 3, e3207. https://doi.org/10.4236/oalib.1103207

[2] Domar, E.D. (1946) Capital Expansion, Rate of Growth, and Employment. Econometrica, 14, 137-147. https://doi.org/10.2307/1905364

[3] Solow, R.M. (1956) A Contribution to the Theory of Economic Growth. The Quar- 
terly Journal of Economics, 70, 65-94. https://doi.org/10.2307/1884513

[4] Abramovitz, M. (1956) Resource and Output Trends in the United States since 1870. American Economic Review, 46, 5-23.

[5] Kaldor, N. (1963) Capital Accumulation and Economic Growth. MacMillan, London.

[6] Denison, E.F. (1974) Accounting for United States Economic Growth, 1929-1969. The Brookings Institution, Washington DC.

[7] Maddison, A. (1987) Growth and Slowdown in Advanced Capitalist Economies: Techniques of Quantitative Assessment. Journal of Economic Literature, 25, 649698.

[8] Jorgenson, D.W., Gollop, F.M. and Fraumeni, B.M. (1987) Productivity and U.S. Economic Growth. Harvard University Press, Cambridge.

[9] Summers, R. and Heston, A. (1991) The Penn World Table (Mark 5): An Expanded Set of International Comparisons, 1950-1988. Quarterly Journal of Economics, 106, 327-368. https://doi.org/10.2307/2937941

[10] Barro, R.J. (1998) Determinants of Economic Growth: A Cross-Country Empirical. MIT Press, Cambridge.

[11] Romer, P.M. (1986) Increasing Returns and Long-Run Growth. Journal of Political Economy, 94, 1002-1037.

[12] Barro, R.J. (1990) Government Spending in a Simple Model of Endogenous Growth. Journal of Political Economy, 98, 103-125. https://doi.org/10.1086/261726

[13] Rebelo, S.T. (1991) Long-Run Policy Analysis and Long-Run Growth. Journal of Political Economy, 99, 500-521. https://doi.org/10.1086/261764

[14] Zhan, M.A. and Zhan, Z. (2016) A Kind of Neither Keynesian Nor Neoclassical Model (2): The Business Cycle. Open Access Library Journal, 3, e3215. https://doi.org/10.4236/oalib.1103215

[15] Zhan, M.A. and Zhan, Z. (2017) A Kind of Neither Keynesian Nor Neoclassical Model (3): The Inflation Equation. Open Access Library Journal, 4, e3333.

[16] Zhan, M.A. and Zhan, Z. (2017) A Kind of Neither Keynesian Nor Neoclassical Model (4): The Nature of Philips Curve. Open Access Library Journal, 4, e3388. https://doi.org/10.4236/oalib.1103388

[17] Chiang, A.C. and Wainwright, K. (1984) Fundamental Methods of Mathematical Economics. 4th Edition, McGraw-Hill, New York.

[18] Arrow, K.J. (1962) The Economic Implications of Learning by Doing. Review of Economic Studies, 29, 155-173. https://doi.org/10.2307/2295952

[19] Lucas, R.E. (1988) On the Mechanics of Economic Development. Journal of Monetary Economics, 22, 3-42. 
Submit or recommend next manuscript to OALib Journal and we will provide best service for you:

- Publication frequency: Monthly

- 9 subject areas of science, technology and medicine

- Fair and rigorous peer-review system

- Fast publication process

- Article promotion in various social networking sites (LinkedIn, Facebook, Twitter, etc.)

- Maximum dissemination of your research work

Submit Your Paper Online: Click Here to Submit

Or Contact service@oalib.com 